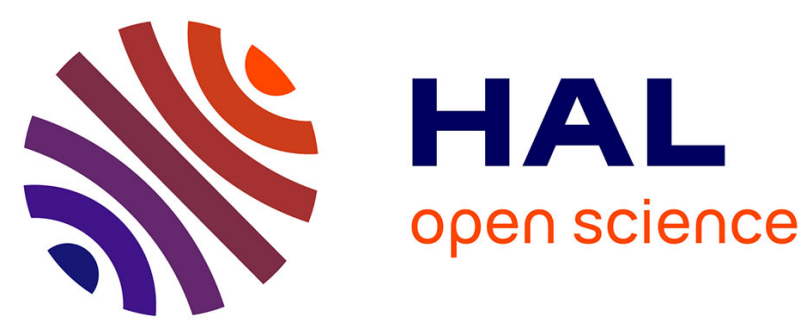

\title{
White Matter Information Flow Mapping from Diffusion MRI and EEG
}

Samuel Deslauriers-Gauthier, Jean-Marc Lina, Russell Butler, Kevin

Whittingstall, Pierre-Michel Bernier, Rachid Deriche, Maxime Descoteaux

\section{- To cite this version:}

Samuel Deslauriers-Gauthier, Jean-Marc Lina, Russell Butler, Kevin Whittingstall, Pierre-Michel Bernier, et al.. White Matter Information Flow Mapping from Diffusion MRI and EEG. NeuroImage, 2019, 10.1016/j.neuroimage.2019.116017 . hal-02187859

\section{HAL Id: hal-02187859 \\ https://hal.inria.fr/hal-02187859}

Submitted on 18 Jul 2019

HAL is a multi-disciplinary open access archive for the deposit and dissemination of scientific research documents, whether they are published or not. The documents may come from teaching and research institutions in France or abroad, or from public or private research centers.
L'archive ouverte pluridisciplinaire HAL, est destinée au dépôt et à la diffusion de documents scientifiques de niveau recherche, publiés ou non, émanant des établissements d'enseignement et de recherche français ou étrangers, des laboratoires publics ou privés. 


\title{
White Matter Information Flow Mapping from Diffusion MRI and EEG
}

\author{
Samuel Deslauriers-Gauthier, ${ }^{\mathrm{a}, *}$, Jean-Marc Lina ${ }^{\mathrm{b}}$, Russell Butler ${ }^{\mathrm{c}}$, Kevin Whittingstall ${ }^{\mathrm{c}}$, Pierre-Michel \\ Bernier $^{c}$, Rachid Deriche ${ }^{\mathrm{a}}$, Maxime Descoteaux ${ }^{\mathrm{c}}$ \\ ${ }^{a}$ Inria Sophia Antipolis-Meditérranée, Université Côte d'Azur, France \\ ${ }^{b}$ École de Technologie Supérieure, Montréal, Canada \\ ${ }^{c}$ Université de Sherbrooke, Sherbrooke, Canada
}

\begin{abstract}
The human brain can be described as a network of specialized and spatially distributed regions. The activity of individual regions can be estimated using electroencephalography and the structure of the network can be measured using diffusion magnetic resonance imaging. However, the communication between the different cortical regions occurring through the white matter, coined information flow, cannot be observed by either modalities independently. Here, we present a new method to infer information flow in the white matter of the brain from joint diffusion MRI and EEG measurements. This is made possible by the millisecond resolution of EEG which makes the transfer of information from one region to another observable. A subject specific Bayesian network is built which captures the possible interactions between brain regions at different times. This network encodes the connections between brain regions detected using diffusion MRI tractography derived white matter bundles and their associated delays. By injecting the EEG measurements as evidence into this model, we are able to estimate the directed dynamical functional connectivity whose delays are supported by the diffusion MRI derived structural connectivity. We present our results in the form of information flow diagrams that trace transient communication between cortical regions over a functional data window. The performance of our algorithm under different noise levels is assessed using receiver operating characteristic curves on simulated data. In addition, using the well-characterized visual motor network as grounds to test our model, we present the information flow obtained during a reaching task following left or right visual stimuli. These promising results present the transfer of information from the eyes to the primary motor cortex. The information flow obtained using our technique can also be projected back to the anatomy and animated to produce videos of the information path through the white matter, opening a new window into multi-modal dynamic brain connectivity.
\end{abstract}

Keywords: dynamic connectivity, functional connectivity, structural connectivity, EEG, diffusion MRI, MEG, brain networks

\footnotetext{
${ }^{*}$ Corresponding author at: Inria Sophia Antipolis-Meditérranée, Université Côte d'Azur, France. Email address: samuel.deslauriers-gauthier@inria.fr
} 


\section{Introduction}

The human brain can be segmented into specialized functional regions (Thirion et al., 2014). These regions are interconnected by white matter fiber bundles which act as information highways. By transferring information to and from different areas, the white matter connections allow the brain to process and respond to external stimuli. Consider the common task of seeing an object and grasping it. Visual information must first be transfered from the eyes to the visual cortex. The limited number of pathways connecting the eyes and the visual cortex leads us to infer that it is likely that information is transferred through the optic nerve, optic tract, and optic radiations. Having reached the occipital lobe, relevant visual information is then thought to be relayed to neural assemblies within parietal, premotor, and motor cortical regions to plan and execute the arm movement(Gallivan and Culham, 2015). Here, the information path is difficult to infer as many white matter pathways connect these cortical regions and there are no non-invasive methods to disambiguate the situation. Our inability to identify the pathways involved is critical as information exchange is fundamental to higher brain functions. Individually, the visual, parietal, premotor, and primary motor cortices cannot achieve the desired task. Indeed, it is the interaction, or more specifically the exchange of information between the nodes of the motor network, that underlie efficient motor behavior. The ability to infer and observe the path taken by the information in the brain would therefore be invaluable to improving our understanding of the structure-function relationship.

The notion of exchange of information or information flow between two cortical regions is closely linked to the concept of connectivity. Structural connectivity refers to the existence of a physical link between two regions that allows the exchange of information. Functional connectivity refers to the statistical relationship between the temporal signals of two anatomically distinct cortical regions (Friston, 1994). While structural connectivity is generally regarded as static over short time periods, functional connectivity changes over time, giving rise to the notion of dynamic functional connectivity. Connectivity, both structural and functional, is required for information exchange, but is not sufficient (Preti et al., 2017). Indeed, the direct transfer of information between two cortical regions requires the existence of a white matter connection between them. However, this connection must also have physical properties coherent with the observed delay in their temporal signals (Horowitz et al., 2015). Additionally, information transfer from one region to another implies an order or directionality that is not necessarily captured by functional connectivity, although some approaches can estimate it (Brovelli et al., 2004). To infer information flow and its path in the white matter, we must then quantify the directed dynamical functional connectivity whose delays are supported by the structural connectivity.

The white matter fiber bundles which support the communication between cortical regions can be imaged using diffusion magnetic resonance imaging (MRI) on a macroscopic scale. However, the information that is assumed to propagate through these fiber bundles cannot be observed with current diffusion MRI technology. More generally, it is impossible to determine if fiber bundles are afferent, efferent, or both using diffusion MRI alone (Jbabdi and Johansen-Berg, 2011). Other acquisitions methods such as electroencephalography (EEG), 
magnetoencephalography (MEG), and functional MRI measure, either directly or indirectly, information processing in the brain but not its path in the white matter. An intuitive approach to quantify information flow is therefore to combine diffusion MRI with functional MRI, EEG, or MEG. Indeed, the joint use of diffusion and functional MRI has received considerable attention (Hutchisson et al., 2013; Zhu et al., 2014; Scaccianoce et al., 2016). For example, Jbabdi et al. (2007) propose a global tractography algorithm which can include functional connectivity information. Calamante et al. (2013) present a method to project functional MRI data onto the white matter substrate supporting it. However, because of the limited temporal resolution of functional MRI, the information flow which occurs at the millisecond scale is blurred into a single volume and cannot be resolved. The joint use of diffusion MRI and M/EEG has been investigated to parcellate the cortex in the context of the inverse problem (Philippe et al., 2012; Belaoucha et al., 2014, 2015, 2016; Belaoucha and Papadopoulo, 2017). Their joint use in the context of connectivity has also been investigated (Sui et al., 2013; Hutchisson et al., 2013), but most studies focus on their combination rather than their fusion (Westerhausen et al., 2006; Tertel et al., 2011; Horowitz et al., 2015). While these techniques do make use of joint structural and functional information, they do not attempt to model or regularize the dynamics of EEG using diffusion MRI. One notable exception is the work of Fukushima et al. (2015) which makes use of diffusion and functional MRI to constrain the resolution of the MEG inverse problem. In their work, source intensities are modeled using a multivariate autoregressive model where diffusion MRI is used to initialize the model coefficients. Given MEG data, the posterior distribution of the coefficients is then estimated using a variational Bayesian algorithm. By observing the resulting coefficients, the authors quantify directed interaction between cortical sources. However, because the coefficients of the model are static, this model cannot accommodate transient information flow between different cortical regions. A multivariate autoregressive model using an alternative minimization procedure was also proposed by Belaoucha and Papadopoulo (2016, 2017). David et al. (2006) suggest the use of dynamical causal modeling (Friston et al., 2003) to constrain cortical source reconstruction in EEG, but make use of prior knowledge rather that diffusion data to generate the constraints. These techniques make use of the complementary nature of the EEG and dMRI signals to recover a static measure of connectivity informed by both structural and functional data. None of them allow the estimation of the directed dynamical functional connectivity between cortical regions supported by structural connectivity.

In this work, we propose the joint use of EEG and diffusion MRI to non-invasively infer and visualize the flow of information through the white matter that connects brain regions. This is made possible by the millisecond temporal resolution of EEG, making the inter-cortical region communication have an observable delay. This is in stark contrast to the temporal resolution of functional MRI where the communication would appear instantaneous. Compared to the techniques discussed above, our approach models connections as the time-varying relationships between cortical regions. This allows us to not only estimate which connections are active but also at which moment over the data window. In addition, because the connections between brain regions are derived from diffusion MRI tractography, the connection activity can be projected back to the 
anatomy and animated to produce videos of the information path through the white matter. Our approach is built on the previous work of Amblard et al. (2004), with several modeling and methodological differences which will be highlighted in the following sections. We refer to our new algorithm as Connectivity Informed Maximum Entropy on the Mean (CIMEM). Preliminary results of this work have previously been published (Deslauriers-Gauthier et al., 2017) but with limited information on the method and its evaluation. Here, we provide a detailed description of our inference algorithm and evaluate its performance using receiver operating characteristic curves on simulated data. In addition, using the well-characterized visual motor network as grounds to test our model, we present the information flow obtained during a reaching task following left or right visual stimuli. In addition to videos provided in the supplementary materials, our results are reported using information flow diagrams which illustrate the transfer of information through the white matter on a millisecond scale.

\section{Theory}

At the heart of the CIMEM model is a Bayesian network which encapsulates our prior knowledge of the brain's state. This prior knowledge, which cover connections, cortical regions, and cortical activity, is related to the EEG measurements through the forward model. Together, the Bayesian network and the forward model constitute the generative model. By setting the state of the hidden variables of the network that describe which cortical regions and connections are active, it generates source activity and thus EEG measurements. More interestingly, this model can be inverted and thus provide information on possible brain states given EEG recordings. Furthermore, because the white matter connections are intrinsic to the model, we are able to infer which of them are used to transfer information through the brain. The following subsections elaborate on this generative model starting from the EEG forward model and moving up to cortical source, regions, and connections. We then present how it can be inverted to obtain information flow in the white matter.

\subsection{Forward model}

We model the cortical activity using a distributed dipole model (Baillet et al., 2001). The activation of a small area of the cortical surface is modeled by a dipole whose orientation is fixed perpendicular to the cortical surface. The intensity of the $n^{\text {th }}$ dipole at a time $t$ is given by $x_{n, t} \in \mathbb{R}$. We distribute several thousands of these dipoles on the cortical surface and combine their intensities in a vector $\boldsymbol{x}_{t}=\left[x_{1, t}, x_{2, t}, \ldots, x_{N, t}\right]$, where $\mathrm{N}$ is the number of dipoles. The EEG measurements at an instant $t$ can then be modeled as

$$
\boldsymbol{m}_{t}=\tilde{\boldsymbol{G}} \boldsymbol{x}_{t}+\boldsymbol{\epsilon}_{t}
$$

where $\boldsymbol{m}_{t}=\left[\begin{array}{llll}m_{1, t} & m_{2, t} & \cdots & m_{M, t}\end{array}\right]^{T}$ is the vector of EEG measurements with $M$ the number of sensors, typically between 60 and 300. The matrix $\tilde{G} \in \mathbb{R}^{M \times N}$ is the lead field that projects the dipole intensities onto the sensors. It can be computed using the anatomical T1-weighted MRI of the subject (Gramfort et al., 2010) and is therefore a known quantity. The observations are corrupted by the additive noise term $\boldsymbol{\epsilon}_{t} \in \mathbb{R}^{M}$. 


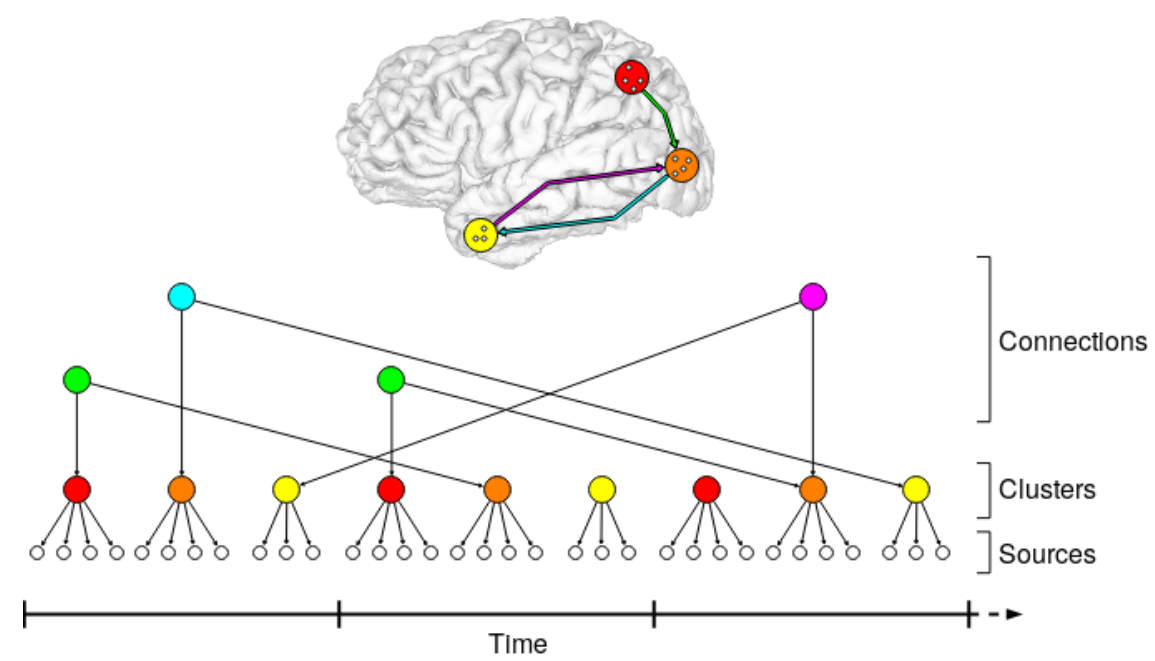

Figure 1: The Bayesian network used to model cortical activity of a simplified brain model. The top panel presents the anatomical model which contains 11 sources illustrated by the small white circles at each time instant, 3 source clusters illustrated by the red, orange, and yellow larger circles, and 3 connections illustrated by the green, teal, and pink arrows. The bottom panel presents the associated Bayesian network truncated to 3 times samples. The green connection affects the red and orange clusters with a delay of 1 , whereas the teal and pink connections affect the orange and yellow clusters with a delay of 2 . Notice that the connectivity between the yellow and the orange clusters is bidirectional and is thus modeled using 2 independent connections (teal and pink).

While the forward model of Eq. (1) provides the EEG measurements associated with source intensities, a more practical situation is to estimate the cortical source intensities associated with EEG measurements, that is, solving the inverse problem. Estimating these intensities from the measurements $\boldsymbol{m}$ is challenging because we typically have many more dipoles than sensors, making the problem ill-posed. In many algorithms, these intensities are computed using an inverse operator $\tilde{\boldsymbol{G}}^{-1}$ which may include regularization (Pascual-Marqui et al., 1994; Hämäläinen and Ilmoniemi, 1994). The dipole intensities are then estimated with $\tilde{\boldsymbol{x}}_{t}=\tilde{\boldsymbol{G}}^{-1} \boldsymbol{m}_{t}$. In this situation, it is convenient to concatenate all measurements $\boldsymbol{m}_{t}$ with $t=1,2, \ldots, T$ in a matrix with a shape $M \times T$. The dipole intensities can then be estimated using a single matrix product. We choose an alternate route and consider all time samples in a single very large problem written as

$$
\boldsymbol{m}=\boldsymbol{G x}+\boldsymbol{\epsilon}
$$

where $\boldsymbol{m} \in \mathbb{R}^{M T}$ and $\boldsymbol{x} \in \mathbb{R}^{N T}$ are the concatenation of all measurements and dipole intensities, respectively. The new forward operator $\boldsymbol{G} \in \mathbb{R}^{M T \times N T}$ is block diagonal and contains copies of $\tilde{\boldsymbol{G}}$ on its diagonal. Despite the very large size of $\boldsymbol{G}$, the problem remains tractable because our approach does not require $\boldsymbol{G}^{-1}$ and the sparse nature of $\boldsymbol{G}$ allows us to implement products efficiently. This formulation allows us to treat a data window of arbitrary size as a single problem which simplifies the notation by dropping the time indexes. In addition, because all data samples are considered at once, a temporal regularization may be introduced. For the remainder of the paper, $\boldsymbol{m}$ and $\boldsymbol{x}$ will therefore refer to the EEG measurements and the source intensities of a time window of arbitrary size. 


\subsection{Maximum Entropy on the Mean}

The vector $\boldsymbol{x}$ contains the source intensities which define the cortical activity over a time window. Let $d \mu(\boldsymbol{x})=\mu(\boldsymbol{x}) d \boldsymbol{x}$ be a reference law that represents the a priori information of the dipole intensities. That is, given some source intensities $\boldsymbol{x}, d \mu(\boldsymbol{x})$ provides the likelihood of this configuration. By specifying $d \mu(\boldsymbol{x})$, we can make certain source intensity configurations more likely than others and thus favor certain solutions when estimating cortical activity from EEG measurements.

Because sources in a distributed model represent a small portion of the cortical surface, it is reasonable that neighboring sources will have correlated intensities. This implies spatial correlation or regularization of the source intensities on the cortical surface. One way to add this spatial regularity to the sources is by defining cortical regions, as suggested by Amblard et al. (2004). These cortical regions can be defined using an atlas (Desikan et al., 2006), be data driven (Thirion et al., 2014), structural connectivity driven (Gallardo et al., 2018), or use a multi-modal approach (Glasser et al., 2016). Regardless of the selected parcellation strategy, the cortical surface is divided in $N_{S}$ cortical regions and each cluster is assigned a state variable which dictates the possible intensities of dipoles within the cluster. For example, we can define clusters to have two states: active and inactive. If a cluster is active, then the intensities of the sources within this cluster follow a Gaussian distribution with nonzero mean whereas if the cluster is inactive, the intensities are zero. Let $S_{k}$ represent the state of the $k^{t h}$ cluster and $S=\left[S_{1}, S_{2}, \ldots, S_{N_{S}}\right]$ be the cluster state vector. Note that like $\boldsymbol{m}$ and $\boldsymbol{x}$, the vector $\boldsymbol{S}$ incorporates a time component. The state $S_{k}$ therefore refers to a specific cortical region at a specific time and the same region at different time would bear a different index. The probability of observing a given source intensity configuration $\boldsymbol{x}$ and cortical cluster configuration $\boldsymbol{S}$ is given by

$$
d \mu(\boldsymbol{x}, \boldsymbol{S})=\pi(\boldsymbol{S}) d \mu(\boldsymbol{x} \mid \boldsymbol{S})
$$

where $\pi(\boldsymbol{S})$ is the joint probability law of the cluster states. Analogously to $d \mu(\boldsymbol{x})$ for source intensities, $\pi(\boldsymbol{S})$ gives the likelihood of observing a cluster configuration $\boldsymbol{S}$. The distribution in Eq. (2) is very general. It assumes the activity of a source can be influenced by the state of any or all clusters. In practice, it is unlikely that the state of a dipole will be directly affected by a distant cortical surface. The reference law of Eq. (2) may therefore be simplified without compromising its realistic feature. We assume the source amplitudes $\boldsymbol{x}_{k}$ of the $k^{\text {th }}$ cluster depends only on the state of the cluster $S_{k}$ to get

$$
d \mu(\boldsymbol{x} \mid \boldsymbol{S})=\prod_{k=1}^{N_{S}} d \mu\left(\boldsymbol{x}_{k} \mid S_{k}\right)
$$

which can be inserted into Eq. (2) to yield

$$
d \mu(\boldsymbol{x}, \boldsymbol{S})=\pi(\boldsymbol{S}) \prod_{k=1}^{N_{S}} d \mu\left(\boldsymbol{x}_{k} \mid S_{k}\right)
$$

In Amblard et al. (2004), it was further assumed that cluster states were independent and the resulting distribution was used to constrain the inverse problem and estimate the dipole intensities in a technique 
called Maximum Entropy on the Mean (MEM). In Connectivity Informed MEM, a dependence between cluster states is expected and will be used to model information flow between cortical regions.

\subsection{Connectivity Informed Maximum Entropy on the Mean (CIMEM)}

The distribution in Eq. (3) regularizes the inverse problem by adding a spatial constraint on the dipoles in the form of cluster states. Here, we propose to modify this distribution to add the structural connectivity information obtained from diffusion MRI. In other words, we propose to further regularize the inverse problem by adding a temporal constraint based on subject specific structural connectivity.

The output of a tractography pipeline is a set of streamlines which represent the white matter fiber bundles. When streamlines begin in a region and end in another, we say that those two regions are connected. While we will mostly be concerned with cortico-cortical connections, this definition applies to other structures e.g. the eyes are connected to the lateral geniculate nucleus by the optic nerve and optic tract. Because information is transferred between cortical regions via connections, clusters that are connected can influence each other. For example, if a cluster is active at a time $t_{0}$, it increases the likelihood that a second cluster is active at a time $t_{\Delta}$. The delay $\Delta$ depends on the characteristic of the connection which include its length, axon diameter, and myelin thickness. This temporal regularization between clusters constitutes an additional prior and is captured by modifying Eq. (3) to include a new set of variables which represent connection states. The state of a connection $C_{i}$ gives us information about the state of the clusters it connects. Let $\boldsymbol{C}$ be the connection state vector, the modified probability of observing a given brain state is then given by

$$
d \mu(\boldsymbol{x}, \boldsymbol{S}, \boldsymbol{C})=\varphi(\boldsymbol{C}) \pi(\boldsymbol{S} \mid \boldsymbol{C}) \prod_{k=1}^{N_{S}} d \mu\left(\boldsymbol{x}_{k} \mid S_{k}\right)
$$

where $\varphi(\boldsymbol{C})$ is the joint probability law of the connection states. Again, the distribution in Eq. (4) is too general because it allows a connection to influence clusters it does not reach. To simplify it, we add the constraint that the state of a cluster depends only on the connections that reach it. If we let $\gamma(k)$ be the set of connections that reach the $k^{\text {th }}$ cluster, the joint distribution of the cluster states given the connection states is

$$
\pi(\boldsymbol{S} \mid \boldsymbol{C})=\prod_{k=1}^{N_{S}} \pi\left(S_{k} \mid \boldsymbol{C}_{\gamma(k)}\right) .
$$

Finally, we further simplify the model by assuming independent connection states which, along with Eq. (5) reduces Eq. (4) to

$$
d \mu(\boldsymbol{x}, \boldsymbol{S}, \boldsymbol{C})=\prod_{i=1}^{N_{C}} \varphi\left(C_{i}\right) \prod_{k=1}^{N_{S}} \pi\left(S_{k} \mid \boldsymbol{C}_{\gamma(k)}\right) d \mu\left(\boldsymbol{x}_{k} \mid S_{k}\right) .
$$

The distribution Eq. (6) contains all of the subject specific information provided by the T1 weighted and diffusion MRI. Because it describes prior knowledge, it encapsulates everything we know of the brain's state given no EEG measurements. Because the dependencies between connections, clusters, and sources are directed and acyclic, the priors in Eq. (6) can also be encoded as a Bayesian network. A schematic view of the Bayesian network associated with the CIMEM model is illustrated in Figure 1. We are therefore able to 
perform inference on this network and obtain marginal probabilities for any variable in $\boldsymbol{S}$ or $\boldsymbol{C}$. Doing so would yield the prior probability that a cluster or connection is in a given state. However, because we are interested on posterior probabilities given the EEG measurements, the next step is to insert the observed EEG measurements as evidence into the Bayesian network. We do so by using the maximum entropy on the mean approach (Jaynes, 1957).

Let the dipole intensities $\boldsymbol{x}$ have a probability law $d p(\boldsymbol{x})$, distinct from $d \mu(\boldsymbol{x})$ which describes the prior knowledge on $\boldsymbol{x}$. The objective of the maximum entropy on the mean is to find the probability law $d p^{*}(\boldsymbol{x})=$ $p^{*}(\boldsymbol{x}) d \boldsymbol{x}$ that satisfies

$$
\boldsymbol{m}=\boldsymbol{G} \boldsymbol{x}^{*} \text { with } \boldsymbol{x}^{*}=\int \boldsymbol{x} p^{*}(\boldsymbol{x}) d \boldsymbol{x}
$$

while minimizing the distance to the prior knowledge embedded in $d \mu(\boldsymbol{x})$, which is measured using the Kullback-Leibler divergence

$$
D_{K L}(p(\boldsymbol{x}))=\int_{-\infty}^{\infty} p(\boldsymbol{x}) \ln \frac{p(\boldsymbol{x})}{\mu(\boldsymbol{x})} d \boldsymbol{x} .
$$

In words, the maximum entropy on the mean solution is the probability law $d p^{*}(\boldsymbol{x})$ closest to the reference law $d \mu(\boldsymbol{x})$ whose average explains the measurements. Using the method of Lagrange multipliers, minimizing the Kullback-Leibler divergence subject to the constraint in Eq. (7) can be written as

$$
\underset{p(\boldsymbol{x}), \boldsymbol{\lambda}, \lambda_{0}}{\operatorname{minimize}} \mathcal{L}\left(p(\boldsymbol{x}), \boldsymbol{\lambda}, \lambda_{0}\right)
$$

where

$$
\begin{aligned}
\mathcal{L}\left(p(\boldsymbol{x}), \boldsymbol{\lambda}, \lambda_{0}\right) & =D_{K L}(p(\boldsymbol{x})) \\
& -\boldsymbol{\lambda}\left(\boldsymbol{m}-\boldsymbol{G} \int \boldsymbol{x} p(\boldsymbol{x}) d \boldsymbol{x}\right) \\
& -\lambda_{0}\left(1-\int d p(\boldsymbol{x})\right) .
\end{aligned}
$$

The first two terms embody the compromise between the data fit and the relative entropy of $p(\boldsymbol{x})$. The third term ensures $p(\boldsymbol{x})$ is a probability distribution and can be thought of as a scaling coefficient. If we assume the measurement noise is Gaussian with zero mean and with a variance $\boldsymbol{\Sigma}_{\epsilon}^{2}$, the unique optimal Lagrange multipliers $\boldsymbol{\lambda}^{*}$ can be determined by solving (see Appendix A for a sketch of the proof)

$$
\boldsymbol{\lambda}^{*}=\underset{\boldsymbol{\lambda}}{\arg \min } \ln Z(\boldsymbol{\lambda})-\left(\boldsymbol{\lambda}^{T} \boldsymbol{m}-\boldsymbol{\lambda}^{T} \boldsymbol{\Sigma}_{\epsilon}^{2} \boldsymbol{\lambda}\right)
$$

where $Z(\boldsymbol{\lambda})$ is the partition function given by

$$
Z(\boldsymbol{\lambda})=\int \exp \left(\boldsymbol{\lambda}^{T} \boldsymbol{G} \boldsymbol{x}\right) d \mu(\boldsymbol{x}) .
$$

In contrast to Eq. (8), the problem in Eq. (9) does not depend on $p(\boldsymbol{x})$ or $\lambda_{0}$ and is convex with respect to $\boldsymbol{\lambda}$. Indeed, the probability density with maximum entropy $p^{*}(\boldsymbol{x})$ and $\lambda_{0}$ are completely defined by the optimal $\boldsymbol{\lambda}^{*}$. Minimizing Eq. (9) requires evaluating $Z(\boldsymbol{\lambda})$ which in turn requires the problem specific reference law 
$d \mu(\boldsymbol{x})$. In CIMEM, the reference law is obtained by marginalizing $\boldsymbol{S}$ and $\boldsymbol{C}$ out of Eq. (6), that is

$$
d \mu(\boldsymbol{x})=\sum_{\{\boldsymbol{C}\}} \prod_{i=1}^{N_{C}} \varphi\left(C_{i}\right) \sum_{\{\boldsymbol{S}\}} \prod_{k=1}^{N_{S}} \pi\left(S_{k} \mid \boldsymbol{C}_{\gamma(k)}\right) d \mu\left(\boldsymbol{x}_{k} \mid S_{k}\right)
$$

where the sum over $\{\boldsymbol{C}\}$ indicates a sum over all possible realizations of $\boldsymbol{C}$. The partition function is then

$$
Z(\boldsymbol{\lambda})=\sum_{\{\boldsymbol{C}\}} \prod_{i=1}^{N_{C}} \varphi\left(C_{i}\right) \prod_{k=1}^{N_{S}} Z_{k}(\boldsymbol{\lambda})
$$

with

$$
Z_{k}(\boldsymbol{\lambda})=\sum_{\left\{S_{k}\right\}} \pi\left(S_{k} \mid \boldsymbol{C}_{\gamma(k)}\right) \int \exp \left(\boldsymbol{\lambda}^{T} \boldsymbol{G}_{k} \boldsymbol{x}_{k}\right) d \mu\left(\boldsymbol{x}_{k} \mid S_{k}\right)
$$

As stated previously, minimizing Eq. (9) requires evaluating the partition function $Z(\boldsymbol{\lambda})$ repeatedly. The sum over all possible states of $\boldsymbol{C}$ which appears in Eq. (12) is computationally prohibitive because it generates $2^{N_{C}}$ summations which quickly becomes intractable. This problem can be solved by noting that $Z(\boldsymbol{\lambda})$ is the unnormalized sum of the marginal probability of any variable in $\boldsymbol{C}$ or $\boldsymbol{S}$. It can therefore be evaluated using efficient algorithms for exact inference on Bayesian networks such as message passing using a junction tree (Cowell et al., 1999). Furthermore, not all terms of the sum need to be evaluated because the state of a cluster depends only on a few connections. Using a suitable variable elimination order, which depends on the specific connections between clusters, the sum of products can be rearranged to compute $Z(\boldsymbol{\lambda})$ efficiently.

\subsection{Information flow in connections}

The algorithm presented in the previous section provides a way to include the structural information obtained in diffusion MRI into the EEG inverse problem. When compared to the original MEM algorithm, the additional regularization provided by the structural connections will undoubtedly affect the cortical source reconstruction. However, investigating its impact is out of the scope of this work and will be investigated in future work. Instead, we focus on our algorithms ability to infer information flow in the white matter, which is new information not available in EEG or diffusion MRI individually. Specifically, we are interested in the probability that a given connection at a given time was used to transfer information between two cortical clusters, given the EEG measurements. Consider the function

$$
Z^{*}(\boldsymbol{C})=Z\left(\boldsymbol{\lambda}^{*}, \boldsymbol{C}\right)=\prod_{i=1}^{N_{C}} \varphi\left(C_{i}\right) \prod_{k=1}^{N_{S}} Z_{k}\left(\boldsymbol{\lambda}^{*}\right)
$$

obtained by introducing Eq. (6) into Eq. (10) without marginalizing $\boldsymbol{C}$ and evaluating at the optimal $\boldsymbol{\lambda}$. Because $\boldsymbol{\lambda}^{*}$ captures the compromise between the EEG measurements $\boldsymbol{m}$ and the prior distribution $d \mu(\boldsymbol{x})$, we think of $Z^{*}(\boldsymbol{C})$ as the unnormalized posterior joint probability of the connection states given the EEG measurements. To obtain the posterior probability that a given connection is in a given state at a given time, we marginalize all other connections and normalize the result to obtain

$$
Z^{*}\left(C_{i}\right)=\frac{1}{Z\left(\boldsymbol{\lambda}^{*}\right)} \sum_{\{\boldsymbol{C}\} \backslash C_{i}} \prod_{i=1}^{N_{C}} \varphi\left(C_{i}\right) \prod_{k=1}^{N_{s}} Z_{k}\left(\boldsymbol{\lambda}^{*}\right)
$$


where $\{\boldsymbol{C}\} \backslash C_{i}$ indicates a sum over all possible states except $C_{i}$. For example, if a connection $C_{i}$ has two possible states, then $Z^{*}\left(C_{i}\right)$ yield two numbers whose sum is 1 and that indicate the probability of being in either states. A probability value can be computed for each connection state, for each time sample, and for both the afferent and efferent directions provided they were included in the model.

\subsection{Prior distribution and parameters}

Computing the optimal parameters $\boldsymbol{\lambda}^{*}$ from Eq. (9) requires the prior distribution $d \mu(\boldsymbol{x})$, which from Eq. (11) requires $\varphi\left(C_{i}\right), \pi\left(S_{k} \mid \boldsymbol{C}_{\gamma(k)}\right)$, and $d \mu\left(\boldsymbol{x}_{k} \mid S_{k}\right)$ with $k=1, \ldots, N_{S}$ and $i=1, \ldots, N_{C}$. The probability mass function $\varphi\left(C_{i}\right)$ gives the probability of observing a given state for the $i^{\text {th }}$ connection. Because our objective is to estimate the information flow, we give all connections two possible states: active (state 1) if it is transferring information and inactive (state 0) if it is not. Because we are interested in the information flow given the EEG data, we set the prior connection to $\varphi\left(C_{i}=0\right)=0.99$ and $\varphi\left(C_{i}=1\right)=0.01$. Setting the connection priors in this manner means that connections will only be activated if the EEG data supports it. Like connections, clusters are given two possible states: active (state 0) and inactive (state 1). The probability mass function $\pi\left(S_{k} \mid \boldsymbol{C}_{\gamma(k)}\right)$ therefore gives the probability of observing the $k^{\text {th }}$ cluster as active or inactive given the state of the connections that reach it. To transfer information into a connection, a cortical region must be active, and receiving information from a connection activates a region. On the other hand, if all connections that reach a cluster are inactive, the cluster has active probability of $\kappa$. While the values $\kappa$ could be set to zero, using non-zero values allows a cluster to be activated even if its connections are not. Such a situation can occur if connections were missed by the diffusion MRI pipeline or manually excluded and are therefore absent from the model. The value of $\kappa$ is then a measure of the confidence that the model covers all active connections of the brain for the EEG measurements of interest. With these assumptions, the probability mass function of cluster states is

$$
\pi\left(S_{k} \mid \boldsymbol{C}_{\gamma(k)}\right)= \begin{cases}\kappa / F_{k} & \text { if } S_{k}=1 \text { and } C_{i}=0 \forall i \in \gamma(k) \\ \beta / F_{k} & \text { if } S_{k}=1 \text { and } C_{i}=1 \text { for at least one } i \in \gamma(k) \\ \zeta / F_{k} & \text { if } S_{k}=0 \text { and } C_{i}=0 \forall i \in \gamma(k) \\ 0 & \text { otherwise }\end{cases}
$$

where we have empirically selected $\kappa=0.00001, \beta=0.1, \zeta=1$. The constant $F_{k}$ is selected so that the sum of the entries of the probability mass function gives one. Finally, the probability density function $d \mu\left(\boldsymbol{x}_{k} \mid S_{k}\right)$ gives the relative likelihood of observing the source intensities $\boldsymbol{x}_{k}$ given the state $S_{k}$ of the cluster that contains them. We model the source intensities with Gaussian distributions whose mean and variance depend on the cluster state. That is

$$
d \mu\left(\boldsymbol{x}_{k} \mid S_{k}\right)= \begin{cases}A_{0} \exp \left(-\frac{1}{2} \boldsymbol{x}^{T} \boldsymbol{\Sigma}_{0}^{-1} \boldsymbol{x}\right) & \text { if } S_{k}=0 \\ A_{1} \exp \left(-\frac{1}{2}(\boldsymbol{x}-\boldsymbol{\rho})^{T} \boldsymbol{\Sigma}_{1}^{-1}(\boldsymbol{x}-\boldsymbol{\rho})\right) & \text { otherwise }\end{cases}
$$

with $A .=(2 \pi)^{-k / 2}|\boldsymbol{\Sigma} .|^{-1 / 2}$. The mean source intensity of an active region was set to $\boldsymbol{\rho}=\rho \cdot \mathbf{1}$ with $\rho=1 \mathrm{e}-6$. The variance of an active region was set to $\boldsymbol{\Sigma}_{1}=\rho / 4 \cdot \boldsymbol{P} \boldsymbol{P}^{T}$ with $\boldsymbol{P}=\exp (-\boldsymbol{D})$ where $\boldsymbol{D}$ contains the distance 
between the dipoles along the cortical mesh. The variance of an inactive region was set to $\boldsymbol{\Sigma}_{0}=\rho / 20 \cdot \boldsymbol{I}$ where $\boldsymbol{I}$ is the identity matrix. In other words, if a region is active, its dipoles have correlated intensities with nonzero mean whereas if it is inactive, its dipoles are uncorrelated with zero mean. These assumption on source intensities reduces Eq. (13) to (see Appendix B for a sketch of the proof)

$$
\begin{aligned}
Z_{k}(\boldsymbol{\lambda}) & =\pi\left(S_{k}=0 \mid \boldsymbol{C}_{\gamma(k)}\right) \exp \left(\frac{1}{2} \boldsymbol{\lambda}^{t} \boldsymbol{G}_{k} \boldsymbol{\Sigma}_{0}^{2} \boldsymbol{G}_{k}^{T} \boldsymbol{\lambda}\right) \\
& +\pi\left(S_{k}=1 \mid \boldsymbol{C}_{\gamma(k)}\right) \exp \left(\boldsymbol{\lambda}^{t} \boldsymbol{G}_{k} \boldsymbol{\rho}+\frac{1}{2} \boldsymbol{\lambda}^{t} \boldsymbol{G}_{k} \boldsymbol{\Sigma}_{1}^{2} \boldsymbol{G}_{k}^{T} \boldsymbol{\lambda}\right) .
\end{aligned}
$$

Because of this closed form solution, the gradient of $Z(\boldsymbol{\lambda})$ in Eq. (13) can also be computed explicitly allowing an efficient use of conjugate gradient descent in the optimization of Eq. (9).

Another parameter of the model that must be specified is the conduction delay for each connection. This delay dictates which clusters are affected by a given connection and therefore alters the topology of the Bayesian network described by Eq. (6). We define the delay of the $k^{\text {th }}$ a connection as $\Delta_{k}=\ell_{k} / v$ where $\ell_{k}$ is the average length of the streamlines associated with the connection and $v$ is the information conduction speed. We assume that the information conduction speed is constant across the brain and equal to $6 \mathrm{~m} / \mathrm{s}$ (Hursh, 1939; Fukushima et al., 2015) which would correspond to an average axon diameter of $1 \mu \mathrm{m}$. This assumption will be discussed further later and could, in a future generation of our approach, become a variable of the model to be estimated.

\section{Materials and methods}

\subsection{Data acquisition and preprocessing}

Magnetic resonance (MR) images and electroencephalography (EEG) signals were acquired on a healthy volunteer. The MR images included diffusion weighted images acquired using a split acquisition of fast spinecho signals (SPLICE) sequence (b-value $1000 \mathrm{~s} / \mathrm{mm}^{2}, 64$ directions) and a T1 weighted image. We opted for a SPLICE sequence instead of the more common echo planar imaging to limit artifacts and geometric distortions in the frontal lobe and near the optic nerve. After the MR acquisition, the subject was fitted with a 64 channel actiCAP (Brain Products GmbH) EEG cap and was seated in front of a computer screen in a dark quiet room. He was asked to rest his index finger on the space bar of a computer keyboard and fixate a red circle appearing on the screen. The subject was then asked to reach using his right hand for a circle appearing on the left or right side of the screen. The delay between the visual cue and movement onset, that is the release of the space bar, was recorded for each reach. One run consisted of 50 left and 50 right visual cues randomly interleaved to prevent anticipation. A total of 5 runs were performed. After the EEG acquisition, the subject was returned to the MR scanner to acquire an ultra-short echo time MR sequence used to locate the EEG electrodes as described by Butler et al. (2017). Approval for this study was obtained from the Centre de Sante et de Services Sociaux-Institut Universitaire de Geriatrie de Sherbrooke and Centre Hospitalier Universitaire de Sherbrooke ethics committees. The participant provided written informed consent and all experiments were conducted according to the principles expressed in the Declaration of Helsinki. 
The diffusion weighted images were denoised using non-local means (Descoteaux et al., 2008). They were then resampled to $\mathrm{T} 1$ resolution using cubic spline interpolation and the $\mathrm{T} 1$ was registered to the b0 image using non-linear registration with Advanced Normalization Tools (Avants et al., 2008). Fiber orientation distribution functions were computed using constrained spherical deconvolution of order 8 (Tournier et al., 2007) implemented in dipy ${ }^{1}$ (Garyfallidis et al., 2014). Fiber tracking was performed using probabilistic anatomically constrained particle filter tracking (Girard et al., 2014) also implemented in dipy. This required the segmentation of the brain into white matter, gray matter and cerebrospinal fluid which was performed using FSL FAST (Zhang et al., 2001). The surface of the cortex was extracted and segmented from the T1 weighted image using FreeSurfer and then downsampled to 8000 vertices. For each pair of cortical regions, the streamlines reaching the cortex were extracted and their average length was computed. Because using all of the extracted connections would result in an intractable model, we manually selected a subset of these connections. Directed connections were added between the cortical regions typically identified during a reaching task. These include sub regions of the occipital cortex, parietal cortex, and frontal cortex (Beurze et al., 2007). Two different models were used for the left and right visual cues, the only difference being in the directionality of the inter-hemispheric connections. The specific regions and connections are illustrated in Figure 2 in the form of connectivity matrices. Using the same FreeSurfer surface, the forward operator $\tilde{\boldsymbol{G}}$ was computed using OpenMEEG (Gramfort et al., 2010; Kybic et al., 2005) using a conductivity value of 1.0 in the brain, 1.0 in the skin, and 0.0125 in the skull. The EEG processing was performed using MNE (Gramfort et al., 2013, 2014). The EEG signals were first bandpass filtered $(0.1 \mathrm{~Hz}-50 \mathrm{~Hz})$ and resampled at $100 \mathrm{~Hz}$. Epochs were created starting at visual stimulus and ending $350 \mathrm{~ms}$ after the visual stimulus. Epochs with blink artifacts were automatically detected and excluded from further analysis. The epochs for the left and right visual cues were then averaged to produce one visual evoked potential per condition.

\subsection{Simulations}

To quantify the performance of our reconstruction strategy, data were simulated using our proposed forward model. The objective of these simulations was to evaluate the ability of CIMEM to recover information flow under the assumption that our model is valid. To make the simulations as realistic as possible, we used the anatomy of our subject to simulate EEG measurements. For the connections of the model, the same connections as the left visual were used (top of Figure 2). A connection to activate was randomly selected and the corresponding start and end clusters were identified. At time $t=0$ a source from the start cluster was randomly selected and its intensity set to follow a Gaussian waveform. The neighbors of the sources along the cortical mesh were then selected and their intensity was set to a linearly attenuated Gaussian waveform where the attenuation depends on the distance to the start source. The process was repeated to generate a patch of activity reaching 3 levels of neighbors from the start source. A second patch of activity was generated using a random source from the end cluster and delaying the Gaussian waveform by a delay that depends on the

\footnotetext{
${ }^{1}$ http://nipy.org/dipy/
} 
selected connection. EEG measurements were obtained with the forward model in (1). Zero mean Gaussian noise was added with the standard deviation set to obtain the desired signal to noise ratio (SNR) defined as the variance of the signal divided by the variance of the additive noise. These simulated measurements were then used as input to the CIMEM algorithm to recover the activated connections.

\section{Results}

\subsection{Simulated data}

For the simulations, the performance of the reconstructions was evaluated using receiver operating characteristics (ROC) curves. A ROC curve is obtained by plotting the true positive rate against the false positive rate as a function of a threshold. Here, a connection was considered as a true positive if it was selected to generate the EEG measurements and CIMEM returned a posterior probability of being active above a threshold, i.e. $Z^{*}\left(C_{i}=1\right)>\tau$. The true positive rate is then given by the number of true positives recovered over the number of active connections generated during the simulations as a function of the threshold. A connection was considered a false positive if it was not selected to generate the EEG measurements and CIMEM returned a posterior probability of being active above the threshold. The false positive rate is then given by the number of false positives recovered over the inactive connections generated during the simulations as a function of the threshold.

To evaluate the effect of simultaneous information flow in different connections, simulations were performed by selecting a single connection to generate the data and by selecting two connections. The ROC curves are illustrated in Figure 4 for 200 simulations at SNRs of 1, 2, 5, and 10. At a SNR of 10 and when simulating a single connection, our algorithm achieves a true positive rate of 0.93 and a false positive rate of 0.11. As the SNR is decreased, the true positive rate decreases and the false positive rate increases to reach 0.78 and 0.16 , respectively, at a SNR of 1 . Very similar results are obtained when 2 connections are activated simultaneously.

One representative example of a reconstruction for a SNR of 5 is illustrated in Figure 5 using an information flow diagram. This diagram is constructed by displaying the cortical activation and connection activity as a function of time. For each cortical region, a row of circles is plotted with the color of the circle indicating the probability that the region is active at a given time, i.e. $Z^{*}\left(S_{k}=0\right)$. Lines are then added between the circles if the model contains a connection between those regions. The color of the line represents the probability that the connection is active at a given time. The delay between the start and end point of the lines on the $\mathrm{x}$ axis therefore captures the delay associated with that connection. An information flow diagram can be seen as time varying connectivity matrix where the delays in information flow are accounted for.

\subsection{In vivo data}

Figure 6 illustrates the information flow diagrams obtained for the reaching task following a visual stimulus in the left visual field. In addition to being presented in the form of an information flow diagram, the 
connection activations can also be mapped back to the streamlines obtained in diffusion MRI. Once projected back onto the streamlines as illustrated by Figure 3, they produce a map of information transfer in the brain at a resolution of a few milliseconds. A video representation of the results of Figure 6 is available in the supplementary materials ${ }^{2}$ in addition to still frames presented in the top pane of the figure. Following the left visual field stimulus, information first arrives in the right lateral occipital (LO) cluster from the right lateral geniculate nucleus (LGN) $90 \mathrm{~ms}$ after the stimulus. Information transfer between the right LGN and right LO continues with varying intensity until $160 \mathrm{~ms}$ after the stimulus. Between 140 and $160 \mathrm{~ms}$, the information then flows from the right LO cluster to the left LO cluster through the splenium of the corpus callosum. Nearly simultaneously, both LO regions propagate information to the frontal cortex via the inferior longitudinal fasciculus. Finally and before movement onset, information flows from the frontal cortex to the motor cortex in the left precentral gyrus.

The results for the right visual field stimulus are illustrated in Figure 7 and a video is available in the supplementary materials ${ }^{3}$. A pattern similar to the left visual field stimulus can be identified with a few expected lateralized differences. The information first travels from the left LGN to the left LO cluster $80 \mathrm{~ms}$ after the stimulus and again at $150 \mathrm{~ms}$. As in the left stimulus condition, information then flows from the left LO cluster to the frontal cortex. Simultaneously, information is also transferred intermittently between the left LO and right LO from $150 \mathrm{~ms}$ to $250 \mathrm{~ms}$ after the stimulus. In addition to the main flows described, the connections between the LO regions and the ipsilateral inferior parietal clusters are also sporadically activated for both conditions. Finally, information reaches the left precentral gyrus from the left superior frontal region from $250 \mathrm{~ms}$ to $350 \mathrm{~ms}$.

\section{Discussion}

We presented a new method, coined CIMEM, to infer information flow in the white matter of the brain from joint diffusion MRI and EEG measurements. Within CIMEM, the possible interactions between brain regions at different times are encapsulated in a subject specific Bayesian network derived from diffusion MRI. By introducing EEG measurements as evidence into this model, we are able to infer information flow in white matter connections at each time sample for both the afferent and efferent directions. To visualize these results, we used information flow diagrams which illustrate transient dynamic connectivity between cortical regions at a millisecond resolution. In addition, because each connection corresponds to a collection of streamlines obtained from diffusion MRI tractography, the inferred information flow can also be projected back onto a model of the white matter structural connectivity. When animated, this projection generates a video of the path taken by the information inside the white matter. To our knowledge, CIMEM is the first method that allows the estimation of directed dynamical functional connectivity between cortical regions

\footnotetext{
${ }^{2}$ The left visual field stimulus video is also available here: https://youtu.be/uVatXgtX6ko

${ }^{3}$ The right visual stimulus video is also available here: https://youtu.be/hJtjqrH7CGQ
} 
supported by structural connectivity.

\subsection{In vivo inference of white matter information flow}

CIMEM infers white matter information flow by taking advantage of the joint structure function relation.

For example, the brain regions involved during a reaching task have been well-characterized, but how these regions interact is still an open question. In our in vivo experiments, CIMEM identified cortical regions that are consistent with the previous literature (Beurze et al., 2007) but also provided additional information on their exchange of communication. Specifically, Figures 6 and 7 show that information first reaches the LGN and LO region contra-lateral to the stimulated visual field through the optic nerve, optic tract, and optic radiation. Interhemispheric transfer between the two LO then takes place through the splenium of the corpus callosum. With a delay consistent with the initiation of movement, information is then relayed to the frontal cortex and parietal cortex before reaching the left precental gyrus. Due to the limited number of subjects and the simplified model used, our in vivo result do not allow us to make decisive conclusions on the information transfer during a visual reaching task. Nonetheless, they do serve as a proof of concept for the recovery of white matter information flow which, to the best of our knowledge, cannot be achieved by any other means. Indeed, the transient nature of brain connectivity, even on the scale of hundreds of milliseconds, is well illustrated in the information flow diagrams of Figures 6 and 7. On these time scales, a functional MRI time series would contains a single static image and would be blind to these dynamic connectivity changes. Multivariate autoregressive models making use of diffusion MRI and MEG have sufficient temporal resolution to observe these changes, but by construction assume a stationary connectivity over a data window.

The flexibility of CIMEM approach to model white matter information flow should also be noted. As described here, connections and cortical regions are modeled using two states: active or inactive. This is not an intrinsic limitation of the method and users could choose to model the relationship between connections, cortical regions and sources differently. For example, connections could be modeled to have 3 states: excitatory, inactive, and inhibitory. The posterior probability inferred by CIMEM for each connection would then contain 3 values, one for each state. In general, the proposed framework can accommodate any prior that can be represented as a Bayesian network expressing the relationship between connections, cortical regions, and cortical sources.

\subsection{CIMEM and the M/EEG inverse problem}

As illustrated by the results of Figure 4 obtained on simulated data, CIMEM is able to recover the correct active connections even in the presence of heavy noise. This may be surprising when considering the ill-posed nature of the EEG inverse problem. However, it should be noted that connections are associated with clusters and not individual sources. This allows some leeway in the estimation of the cortical activity as only the correct cortical region needs to be activated to in turn activate the correct connections. Furthermore, because all time samples are considered at once, the posterior distribution of a connection is potentially informed 
by all measurements instead of a single instant. Depending on the anatomical model used, estimating the information flow in the white matter may be a less ill-posed problem than estimating cortical activity.

\subsection{Limitations and future work}

A simplifying assumption used in this work is that all connections share a single constant information conduction velocity equal to $6 \mathrm{~m} / \mathrm{s}$. However, because axon diameter may in fact be subject and bundle specific (Innocenti et al., 2013) and will undoubtedly affect conduction velocities, a more realistic model could make use of white matter bundle specific velocities. In the context of CIMEM, this would simply correspond to a connection specific velocity $v_{k}$ used in the computation of the connection delay $\Delta_{k}=v_{k} \ell_{k}$. Therefore, as whole brain axon diameter information becomes available, this information can readily be included as prior knowledge into CIMEM. In addition to axon diameter, many other factors can influence the conduction velocity of axons including myelin g-ratio (Stikov et al., 2015) and other cell and tissue properties (Stufflebeam et al., 2008; Fields et al., 2015). Considering the variety of axonal features which may affect conduction velocity, one may wish to associate a distribution of velocities instead of a single velocity to each connection. In the version of CIMEM presented here, each connection represents a one to one link between clusters because each connection is associated with a single delay. To accommodate distributions of velocities, connections would have to be associated with several delays and represent a one to many relationship between clusters. This can be achieved by modifying the prior distributions of cluster states $\pi\left(S_{k} \mid \boldsymbol{C}_{\gamma(k)}\right)$ to include more incoming connections. However, there is currently no widely accepted model which maps these features to conduction velocities. In the absence of such a model, we have opted to fall back on the simplest available solution: a single constant conduction velocity for all brain connections.

In this work, we assumed that the conduction velocity is given as prior information to build the Bayesian network. However, because conduction velocity is challenging to measure in vivo, it may be interesting to estimate it using CIMEM. A straight forward way to do this would be to include the same connection multiple times with different delays. The posterior probabilities obtained would then contain information on likely conduction velocities, which may vary from connection to connection. One drawback of this approach is that it greatly increases the number of variables in the system. This leads to both increased numerical complexity and uncertainty in the estimation of the posterior probabilities. Nonetheless, given a well studied paradigm it may be possible to assume a known information flow and limit the number of connections in the model. CIMEM could then be seen as a way to infer the conduction velocities of a few well defined connections.

The Bayesian graph which encodes the prior knowledge used in CIMEM is built using diffusion MRI and dictates the temporal connections that can be active or inactive between different clusters. It is assumed that this graph is a superset of the active connections; it must contain at least all connections used during the functional window. Using the functional information from EEG, CIMEM assigns a posterior probability to each structural connection. Applying a threshold to these posterior probabilities can be understood as a pruning of structural connections to keep only those supported by both anatomical and functional data. 
CIMEM may then provide a way to limit the number of false positive connections in diffusion MRI (MaierHein and Descoteaux, 2017).

The present work focuses on the new information provided by the joint use of diffusion MRI and EEG. In addition, because CIMEM also provides cortical source intensities, it can be described as a solver for the EEG inverse problem. The connectivity information provided by diffusion MRI is an additional temporal constraint and will thus influence the cortical source reconstruction provided by CIMEM. The impact of this additional constraint on source reconstruction will be investigated in future work.

\section{Conclusion}

We have proposed a new method, named CIMEM, to detect information flow using both diffusion MRI and EEG. By using both structural and functional connectivity information, CIMEM is able to infer information flow in the white matter of the brain, information not provided by diffusion MRI or EEG. We hope this pioneering work not only proposed an efficient and mathematically well grounded framework to infer information flow in the white matter from diffusion MRI and EEG but also paves the way for innovative developments in the understanding of the relation between structure and function.

\section{Acknowledgments}

The authors would like to thank Guillaume Gilbert of Phillips for his advise and help in acquiring diffusion MRI data using the SPLICE sequence.

This work has received funding from the European Research Council (ERC) under the European Union's Horizon 2020 research and innovation program (ERC Advanced Grant agreement No 694665 : CoBCoM Computational Brain Connectivity Mapping).

\section{Appendix A. Principle of maximum entropy}

Let $\boldsymbol{x}=\left[x_{1}, x_{2}, \ldots, x_{N}\right]$ be random vector. We are not given the joint distribution $p(\boldsymbol{x})$; all we know are the expected values

$$
E\left(f_{j}(\boldsymbol{x})\right)=m_{j} \text { with } j=1, \ldots, M
$$

Given a distribution $p(\boldsymbol{x})$, its uncertainty, which is synonimus with entropy, is given by

$$
D_{K L}(p(\boldsymbol{x}))=\int_{-\infty}^{\infty} p(\boldsymbol{x}) \ln \frac{p(\boldsymbol{x})}{\mu(\boldsymbol{x})} d \boldsymbol{x}
$$


for some prior distribution $\mu(\boldsymbol{x})$. Using Lagrangian multipliers, the distribution with maximum entropy subject to the constraints (A.1) is obtained by maximizing the functional

$$
\begin{aligned}
\mathcal{L}(p(\boldsymbol{x}))= & -\int_{-\infty}^{\infty} p(\boldsymbol{x}) \ln \frac{p(\boldsymbol{x})}{\mu(\boldsymbol{x})} d \boldsymbol{x} \\
& +\lambda_{0}\left(\int_{-\infty}^{\infty} p(\boldsymbol{x}) d \boldsymbol{x}-1\right) \\
& +\sum_{j=1}^{M} \lambda_{i}\left(\int_{-\infty}^{\infty} f_{j}(\boldsymbol{x}) p(\boldsymbol{x}) d \boldsymbol{x}-m_{j}\right) .
\end{aligned}
$$

The extremum is given by setting the derivative equal to zero

$$
\frac{\partial \mathcal{L}(p(\boldsymbol{x}))}{\partial p(\boldsymbol{x})}=-\ln \frac{p(\boldsymbol{x})}{\mu(\boldsymbol{x})}-1+\lambda_{0}+\sum_{j=1}^{M} \lambda_{i} f_{j}(\boldsymbol{x})=0
$$

which yields

$$
p(\boldsymbol{x})=\mu(\boldsymbol{x}) \exp \left(-1+\lambda_{0}\right) \exp \left(\sum_{j=1}^{M} \lambda_{i} f_{j}(\boldsymbol{x})\right) .
$$

To determine the value of the Lagrange multipliers, we introduce (A.2) into the constraints (A.1) to get

$$
\int_{-\infty}^{\infty} f_{j}(\boldsymbol{x}) \exp \left(-1+\lambda_{0}\right) \exp \left(\sum_{j=1}^{M} \lambda_{j} f_{j}(\boldsymbol{x})\right) d \mu(\boldsymbol{x})=m_{j}
$$

which can be written as

$$
\frac{\partial \ln Z(\boldsymbol{\lambda})}{\partial \lambda_{j}}=m_{j}
$$

where

$$
Z(\boldsymbol{\lambda})=\int_{-\infty}^{\infty} \exp \left(\sum_{j=1}^{M} \lambda_{j} f_{j}(\boldsymbol{x})\right) d \mu(\boldsymbol{x})
$$

and

$$
\ln Z(\boldsymbol{\lambda})=1-\lambda_{0}
$$

The value of the Lagrange multipliers can therefore be determined by solving the M equations in (A.3) or equivalently

$$
\underset{\boldsymbol{\lambda}}{\operatorname{minimize}} \ln Z(\boldsymbol{\lambda})-\boldsymbol{\lambda}^{T} \boldsymbol{m}
$$

\section{Appendix B. Partition function for sources intensities following a normal distribution}

Let $\boldsymbol{x} \in \mathbb{R}^{k}$ represent the source intensities in a cortical regions. The partition function for this region is given by

$$
Z(\boldsymbol{\lambda})=\int \exp \left(\boldsymbol{\lambda}^{T} \boldsymbol{x}\right) \mu(\boldsymbol{x}) d \boldsymbol{x}
$$

and we assume $\mu(\boldsymbol{x}) \sim \mathcal{N}(\boldsymbol{\rho}, \boldsymbol{\Sigma})$. We have

$$
Z(\boldsymbol{\lambda})=A \int \exp \left(\boldsymbol{\lambda}^{T} \boldsymbol{x}\right) \exp \left(-\frac{1}{2}(\boldsymbol{x}-\boldsymbol{\rho})^{T} \boldsymbol{\Sigma}^{-1}(\boldsymbol{x}-\boldsymbol{\rho})\right) d \boldsymbol{x}
$$


with $A=(2 \pi)^{-k / 2}|\boldsymbol{\Sigma}|^{-1 / 2}$. Collecting the terms containing $\boldsymbol{x}$ yields

$$
\begin{aligned}
Z(\boldsymbol{\lambda})= & A \int \exp \left(\boldsymbol{\lambda}^{T} \boldsymbol{\rho}+\boldsymbol{\lambda}^{T}(\boldsymbol{x}-\boldsymbol{\rho})-\frac{1}{2}(\boldsymbol{x}-\boldsymbol{\rho})^{T} \boldsymbol{\Sigma}^{-1}(\boldsymbol{x}-\boldsymbol{\rho})\right) d \boldsymbol{x} \\
= & \exp \left(\boldsymbol{\lambda}^{T} \boldsymbol{\rho}+\frac{1}{2} \boldsymbol{\lambda}^{T} \boldsymbol{\Sigma} \boldsymbol{\lambda}\right) \\
& A \int \exp \left(-\frac{1}{2}(\boldsymbol{x}-\boldsymbol{\rho})^{T} \boldsymbol{\Sigma}^{-1}(\boldsymbol{x}-\boldsymbol{\rho})+\boldsymbol{\lambda}^{T}(\boldsymbol{x}-\boldsymbol{\rho})-\frac{1}{2} \boldsymbol{\lambda}^{T} \boldsymbol{\Sigma} \boldsymbol{\lambda}\right) d \boldsymbol{x} \\
= & \exp \left(\boldsymbol{\lambda}^{T} \boldsymbol{\rho}+\frac{1}{2} \boldsymbol{\lambda}^{T} \boldsymbol{\Sigma} \boldsymbol{\lambda}\right) \\
& \underbrace{A \int \exp \left(-\frac{1}{2}(\boldsymbol{x}-\boldsymbol{\rho}-\boldsymbol{\Sigma} \boldsymbol{\lambda})^{T} \boldsymbol{\Sigma}^{-1}(\boldsymbol{x}-\boldsymbol{\rho}-\boldsymbol{\Sigma} \boldsymbol{\lambda})\right) d \boldsymbol{x}}_{1} \\
= & \exp \left(\boldsymbol{\lambda}^{T} \boldsymbol{\rho}+\frac{1}{2} \boldsymbol{\lambda}^{T} \boldsymbol{\Sigma} \boldsymbol{\lambda}\right) .
\end{aligned}
$$

\section{References}

B. Thirion, G. Varoquaux, E. Dohmatob, J.-B. Poline, Which fmri clustering gives good brain parcellations?, Font. Neurosci. 8 (2014) 1-13.

J. P. Gallivan, J. C. Culham, Neural coding within humain brain areas involved in actions, Current Opinion in Neurobiology 33 (2015) 141-149.

K. J. Friston, Functional and Effective Connectivity in Neuroimaging: A Synthesis, Human Brain Mapping (1994) $56-78$.

M. G. Preti, T. A. W. Bolton, D. Van De Ville, The dynamic functional connectome: State-of-the-art and perspective, NeuroImage 160 (2017) 41-54.

A. Horowitz, D. Barazany, I. Tavor, M. Bernstein, G. Yovel, Y. Assaf, In vivo correlation between axon diameter and conduction velocity in the human brain, Brain Structure and Function 220 (2015) 17771788.

A. Brovelli, M. Ding, A. Ledberg, Y. Chen, R. Nakamura, S. L. Bressler, Beta oscillations in a large-scale sensorimotor cortical network: Directional influences revealed by Granger causality, PNAS 101 (2004) 9849-9854.

S. Jbabdi, H. Johansen-Berg, Tractography - where do we go from here ?, Brain Connectivity 1 (2011) $169-183$.

R. M. Hutchisson, T. Womelsdorf, E. A. Allen, P. A. Bandettini, V. D. Calhoun, M. Corbetta, S. D. Penna, J. H. Duyn, G. H. Glover, J. Gonzalez-Castillo, D. A. Handwerker, S. Keilholz, V. Kiviniemi, D. A. Leopold, F. de Pasquale, M. Sporns, O. Walter, C. Chang, Dynamic functional connectivity: Promise, issues, and interpretations, NeuroImage 80 (2013) 360-378. 
D. Zhu, T. Zhang, X. Jiang, X. Hu, H. Chen, N. Yang, J. Lv, J. Han, L. Guo, T. Liu, Fusing DTI and fMRI data: A survey of methods and applications, NeuroImage 102 (2014) 184-191.

E. Scaccianoce, M. Lagan, F. Baglio, M. Preti, N. Bergsland, P. Cecconi, M. Clerici, G. Baselli, G. Papadimitriou, N. Makris, Combined DTIfMRI Analysis for a Quantitative Assessment of Connections Between WM Bundles and Their Peripheral Cortical Fields in Verbal Fluency, Brain Topography 29 (2016) 814-823.

S. Jbabdi, M. W. Woolrich, J. L. R. Andersson, T. E. J. Behrens, A bayesian framework for global tractography, NeuroImage 37 (2007) 116-129.

F. Calamante, R. A. J. Masterton, J.-D. Tournier, R. E. Smith, L. Willats, D. Raffelt, A. Connelly, Trackweighted functional connectivity (TW-FC): A tool for characterizing the structural-functional connections in the brain, NeuroImage 70 (2013) 199-210.

A. C. Philippe, M. Clerc, T. Papadopoulo, R. Deriche, A nested cortex parcellation combining analysis of MEG forward problem and diffusion MRI tractography, in: IEEE International Symposium on Biomedical Imaging (ISBI 2012).

B. Belaoucha, J. M. Lina, M. Clerc, A. C. Philippe, C. Grova, T. Papadopoulo, Using diffusion mri information in the maximum entropy on mean framework to solve meg/eeg inverse problem, in: 19th International Conference on Biomagnetism (BIOMAG 2014).

B. Belaoucha, J. M. Lina, M. Clerc, T. Papadopoulo, MEM-diffusion MRI framework to solve MEEG inverse problem, in: 23rd European Signal Processing Conference (EUSIPCO 2015).

B. Belaoucha, M. Clerc, T. Papadopoulo, Cortical Surface Parcellation via dMRI Using Mutual Nearest Neighbor Condition, in: IEEE 13th International Symposium on Biomedical Imaging (ISBI 2016), pp. 903-906.

B. Belaoucha, T. Papadopoulo, Spatial regularization based on dMRI to solve EEG/MEG inverse problem, in: 39th Annual International Conference of the IEEE Engineering in Medicine and Biology Society (EMBC 2017).

J. Sui, R. Huster, Q. Yu, J. M. Segall, V. D. Calhoun, Function-structure associations of the brain: Evidence from multimodal connectivity and covariance studies, NeuroImage 102 (2013) 11-23.

R. Westerhausen, F. Kreuder, W. Woerner, R. J. Huster, C. M. Smit, E. Schweiger, W. Wittling, Interhemispheric transfer time and structural properties of the corpus callosum, Neuroscience letters 409 (2006) $140-5$.

K. Tertel, N. Tandon, T. M. Ellmore, Probing brain connectivity by combined analysis of diffusion MRI tractography and electrocorticography., Computers in biology and medicine 41 (2011) 1092-9. 
M. Fukushima, O. Yamashita, T. R. Knösche, M. Sato, MEG source reconstruction based on identification of directed source interactions on whole-brain anatomical networks, NeuroImage 105 (2015) 408-427.

B. Belaoucha, T. Papadopoulo, Iterative two-stage approach to estimate sources and their interactions, in: 20th International Conference on Biomagnetism (BIOMAG 2016).

B. Belaoucha, T. Papadopoulo, Large brain effective network from EEG/MEG data and dMR information, in: 7th International Workshop on Pattern Recognition in NeuroImaging (PRNI 2017).

O. David, S. J. Kiebel, L. M. Harrison, J. Mattout, J. M. Kilner, K. J. Friston, Dynamic causal modeling of evoked responses in EEG and MEG, Neuroimage 30 (2006) 1255-1272.

K. J. Friston, L. Harrison, W. Penny, Dynamic causal modeling, NeuroImage (2003) 1273-1302.

C. Amblard, E. Lapalme, J. M. Lina, Biomagnetic source detection by maximum entropy and graphical models, IEEE transactions on bio-medical engineering 51 (2004) 427-42.

S. Deslauriers-Gauthier, J. M. Lina, R. Butler, P. M. Bernier, K. Whittingstall, R. Deriche, M. Descoteaux, Inference and Visualization of Information Flow in the Visual Pathway using dMRI and EEG, in: MICCAI 2017 Medical Image Computing and Computer Assisted Intervention.

S. Baillet, J. C. Mosher, R. M. Leahy, Electromagnetic brain mapping, IEEE Signal Processing Magazine 18 (2001) $14-30$.

A. Gramfort, T. Papadopoulo, E. Olivi, M. Clerc, OpenMEEG: opensource software for quasistatic bioelectromagnetics, Biomedical engineering online 9 (2010) 45.

R. D. Pascual-Marqui, C. M. Michel, D. Lehmann, Low resolution electromagnetic tomography: A new method for localizing electrical activity in the brain, International Journal of Psychophysiology 18 (1994) $49-65$.

M. S. Hämäläinen, R. J. Ilmoniemi, Interpreting magnetic fields of the brain: minimum norm estimates, Medical \& biological engineering \& computing 32 (1994) 35-42.

R. Desikan, F. Sgonne, B. Fischl, B. Quinn, B. Dickerson, D. Blacker, R. Buckner, A. Dale, R. Maguire, B. Hyman, M. Albert, R. Killiany, An automated labeling system for subdividing the human cerebral cortex on MRI scans into gyral based regions of interest, Neuroimage 31 (2006) 968-980.

G. Gallardo, W. Wells III, R. Deriche, D. Wassermann, Groupwise structural parcellation of the whole cortex: A logistic random effects model based approach, NeuroImage 170 (2018) 307-320.

M. Glasser, T. Coalson, E. Robinson, C. Hacker, J. Harwell, E. Yacoub, K. Ugurbil, J. Andersson, C. Beckmann, M. Jenkinson, S. Smith, D. Van Essen, A multi-modal parcellation of human cerebral cortex, Nature 536 (2016) 171-178. 
E. T. Jaynes, Information theory and statistical mechanics, Physical Review 106 (1957) 620-630.

R. Cowell, A. Dawid, S. Lauritzen, D. Spiegelhalter, Probabilistic Networks and Expert Systems, Springer, 1999.

J. Hursh, Conduction velocity and diameter of nerve fibers, American Journal of Physiology 127 (1939).

R. Butler, G. Gilber, M. Descoteaux, P. M. Bernier, K. Whittingstall, Application of polymer sensitive MRI sequence to localization of EEG electrodes, Journal of Neuroscience Methods 278 (2017) 36-45.

M. Descoteaux, N. Wiest-Daessl, S. Prima, C. Barillot, R. Deriche, Impact of rician adapted non-local means filtering on hardi, in: Medical Image Computing and Computer-Assisted Intervention (MICCAI), pp. $122-130$.

B. B. Avants, C. L. Epstein, M. Grossman, J. C. Gee, Symmetric diffeomorphic image registration with cross-correlation: evaluating automated labeling of elderly and neurodegenerative brain, Medical Image Analysis 12 (2008) 26-41.

J. D. Tournier, F. Calamante, A. Connelly, Robust determination of the fibre orientation distribution in diffusion MRI: Non-negativity constrained super-resolved spherical deconvolution, NeuroImage 35 (2007) $1459-1472$.

E. Garyfallidis, M. Brett, B. Amirbekian, A. Rokem, S. Van Der Walt, M. Descoteaux, I. Nimmo-Smith, Dipy, a library for the analysis of diffusion MRI data, Frontiers in Neuroinformatics 8 (2014).

G. Girard, K. Whittingstall, M. Deriche, R. Descoteaux, Towards quantitative connectivity analysis: reducing tractography biases, NeuroImage 98 (2014) 266-278.

Y. Zhang, M. Brady, S. Smith, Segmentation of brain MR images through a hidden Markov random field model and the expectation-maximization algorithm, IEEE transactions on medical imaging 20 (2001) $45-57$.

S. M. Beurze, F. P. de Lange, I. Toni, W. P. Medencorp, Integration of target and effector information in the human brain during reach planning, J. Neurophysiol 97 (2007) 188-197.

J. Kybic, M. Clerc, T. Abboud, O. Faugeras, R. Keriven, T. Papadopoulo, A common formalism for the integral formulations of the forward EEG problem, IEEE Transactions on Medical Imaging 24 (2005) $12-28$.

A. Gramfort, M. Luessi, E. Larson, D. Engemann, D. Strohmeier, C. Brodbeck, R. Goj, M. Jas, T. Brooks, L. Parkkonen, M. Hmlinen, MEG and EEG data analysis with MNE-Python, Frontiers in Neuroscience 7 (2013). 
A. Gramfort, M. Luessi, E. Larson, D. Engemann, D. Strohmeier, C. Brodbeck, L. Parkkonen, M. Hmlinen, MNE software for processing MEG and EEG data, NeuroImage 86 (2014) 446-460.

G. M. Innocenti, A. Vercelli, R. Caminiti, The diameter of cortical axons depends both on the area of origin and target, Cerebral Cortex 24 (2013) 2178-2188.

N. Stikov, J. S. W. Campbell, T. Stroh, M. Lavelée, S. Frey, J. Novek, S. Nuara, M.-K. Ho, B. J. Bedell, R. F. Dougherty, I. R. Leppert, M. Boudreau, S. Narayanan, T. Duval, J. Cohen-Adad, P. Picard, A. Gasecka, D. Côté, G. B. Pike, In vivo histology of the myelin g-ratio with magnetic resonance imaging, NeuroImage 118 (2015) 397-405.

S. M. Stufflebeam, T. Witzel, S. Mikulski, M. S. Hämäläinen, S. Temereanca, J. J. S. Barton, D. S. Tuch, D. S. Manoach, A non-invasive method to relate the timing of neural activity to white matter microstructural integrity., NeuroImage 42 (2008) 710-716.

R. Fields, D. Woo, P. Basser, Glial regulation of the neuronal connectome through local and long-distant communication, Neuron 86 (2015).

K. Maier-Hein, M. Descoteaux, The challenge of mapping the human connectome based on diffusion tractography, Nature Communications 8 (2017). 

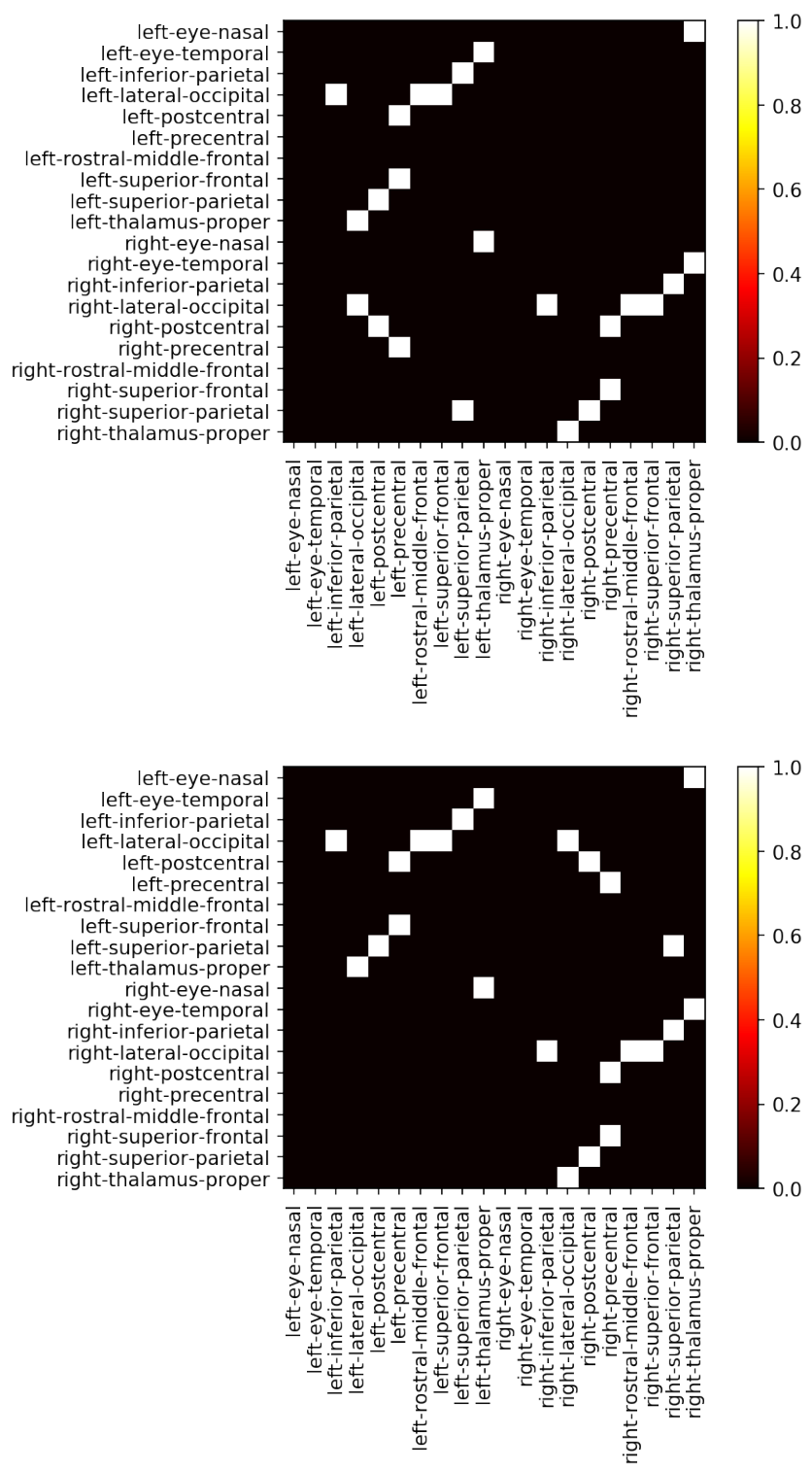

Figure 2: Cortical regions and connections involved in a reaching task with a visual stimulus on the left (top) and on the right (bottom) visual fields. The color indicates the connectivity strength, rows correspond to the starting regions of a connection, and columns correspond to the ending region. Cortical regions with no connections have been removed to simplify the images. The matrices are not symmetric because the connections are directed. 


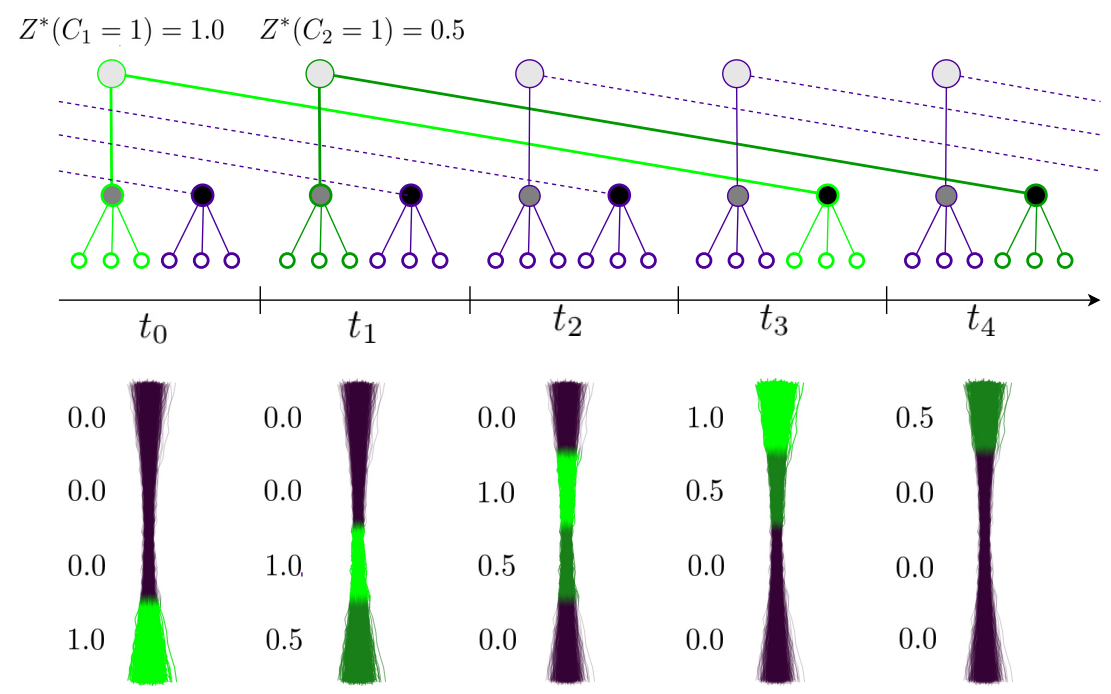

Figure 3: Illustration of the projection of the active probability of connections onto streamlines. The model contains only a single bundle that connects 2 clusters. The posterior probability that the connection is active is 1.0 at $t_{0}, 0.5$ at $t_{1}$, and 0.0 elsewhere. The connections $C_{1}, C_{2}, \ldots$ illustrated in the graph are all time offsets of the same anatomical connection.
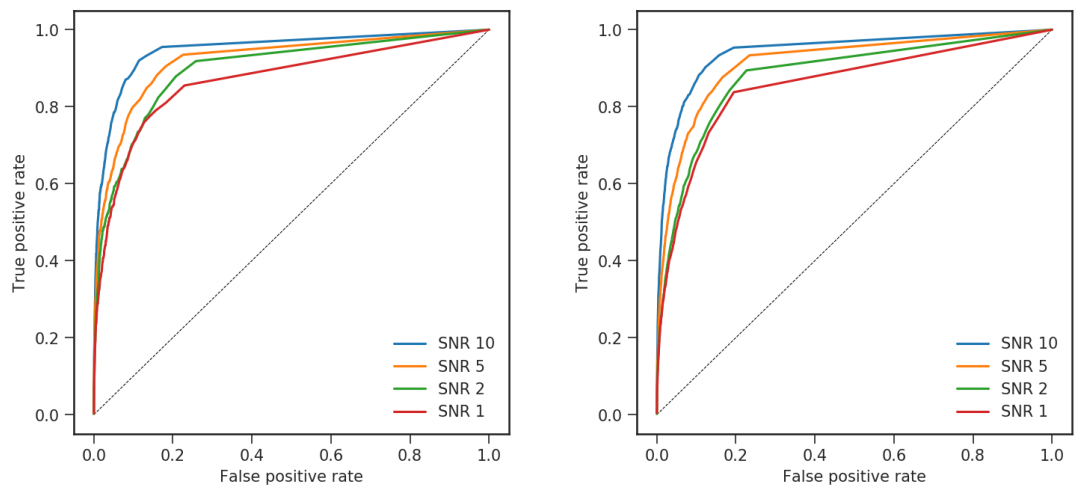

Figure 4: Receiver operating characteristics curves obtained in simulations of 4 SNRs using 1 active connection (left) and 2 active connections (right). 

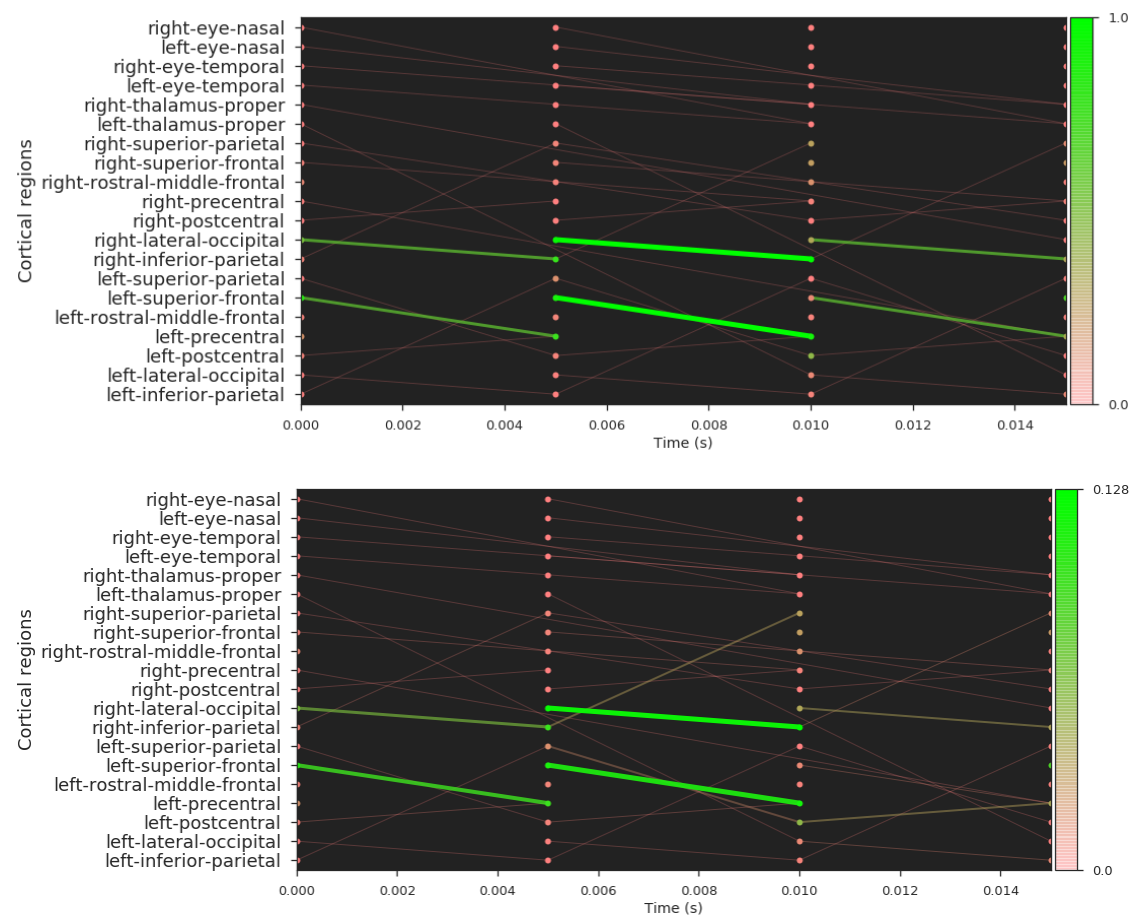

Figure 5: Information flow diagram generated in simulations with an SNR of 5. The top diagram illustrates the simulated information and the bottom one illustrated the recovered flow using CIMEM. Five of the six simulated flows were recovered but the exchange of information between the left superior frontal region and the left precentral gyrus starting at $10 \mathrm{~ms}$ was missed. In addition, two false positives were detected between the right inferior and superior parietal clusters starting at $5 \mathrm{~ms}$ and between the left postcentral and precentral gyri at $10 \mathrm{~ms}$. 

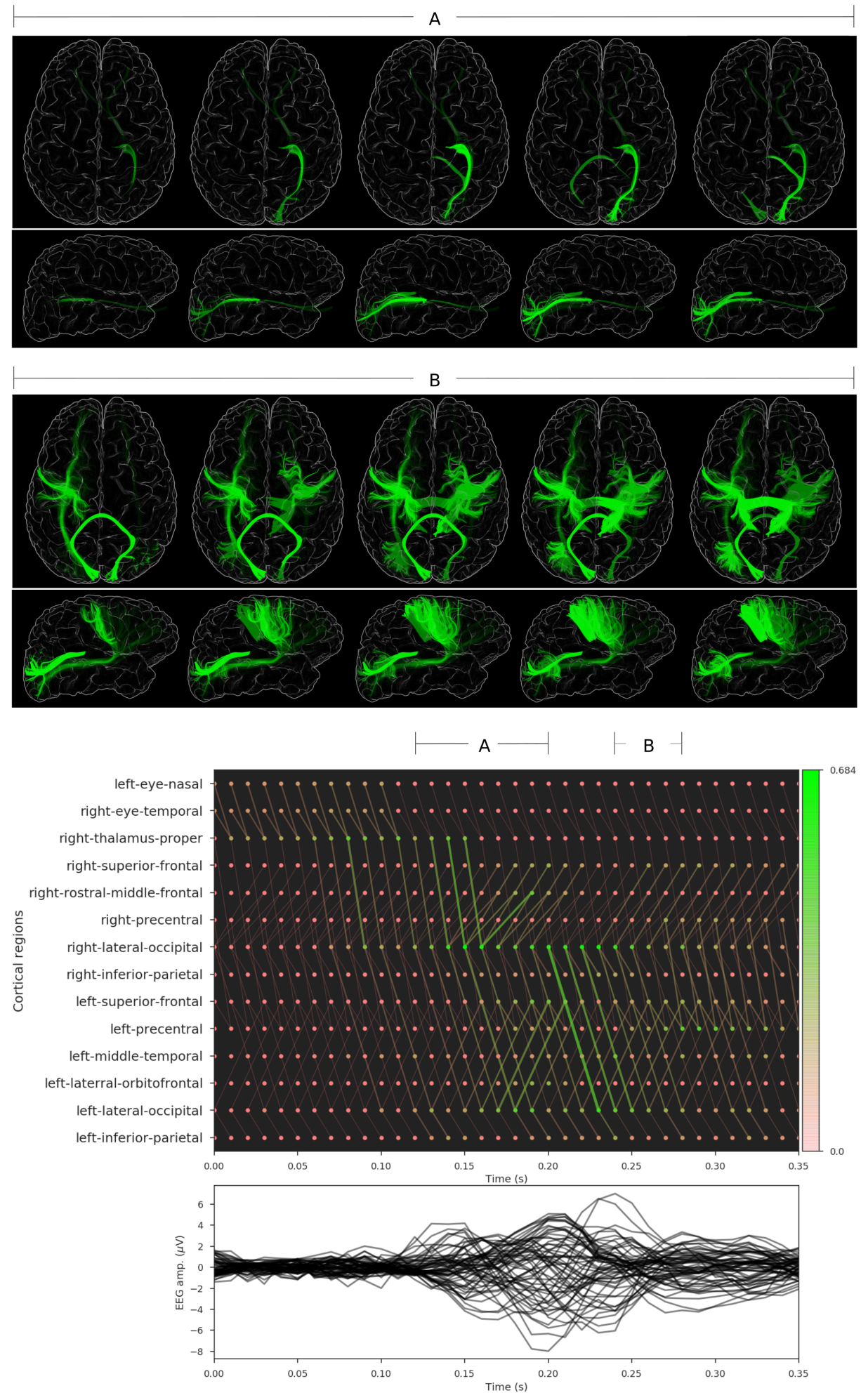

Figure 6: Information flow diagram for a reaching task using the right hand following a visual stimulus on the left visual field. Connections with a probability of being active below 0.15 and clusters with a probability of being active below 0.25 were removed from the graph for clarity. For each diagram, the associated EEG measurements from which they were computed are also presented. The top panels illustrate the information projected back on the anatomy for short data windows. 

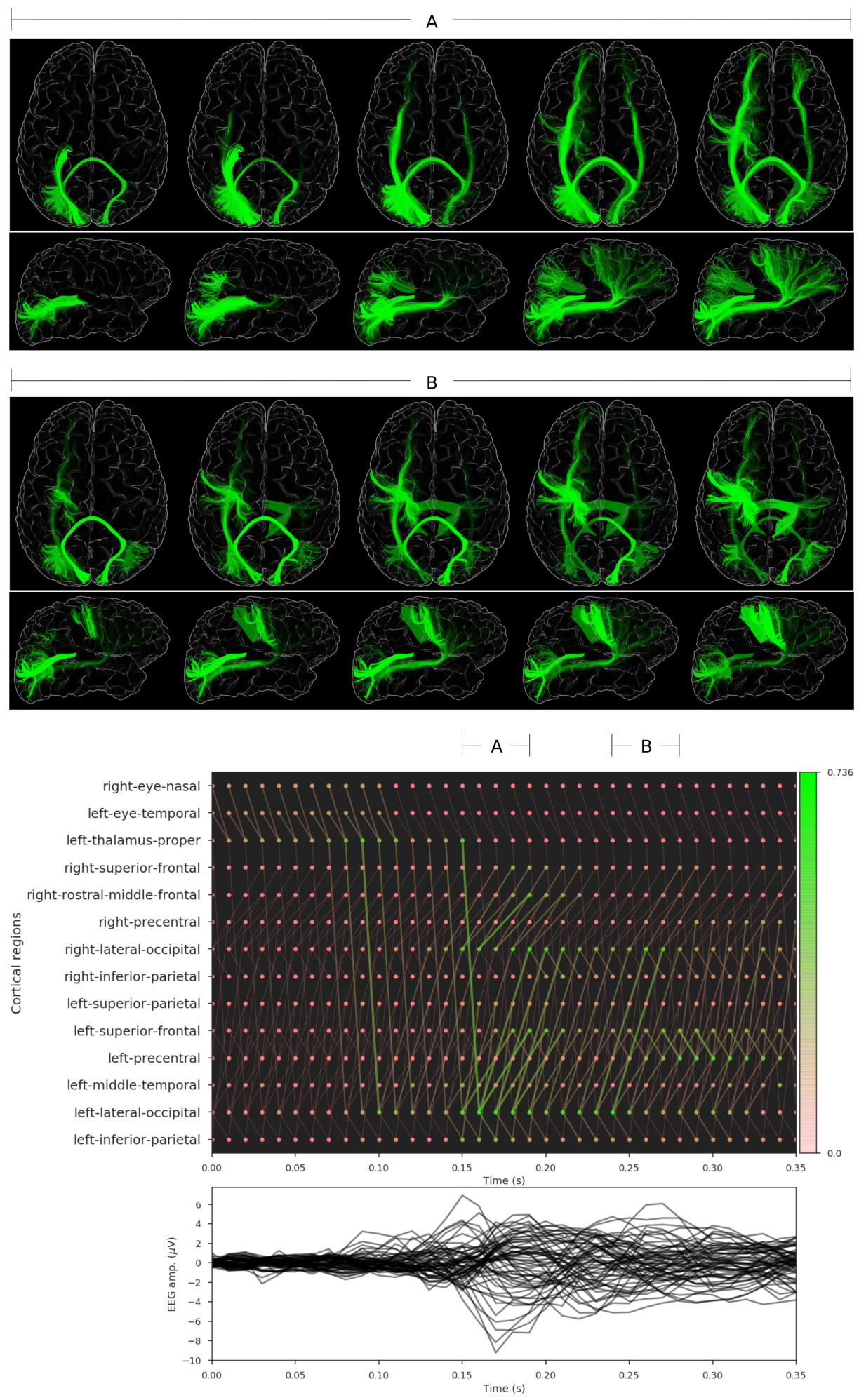

Figure 7: Information flow diagram for a reaching task using the right hand following a visual stimulus on the right visual field. Connections with a probability of being active below 0.15 and clusters with a probability of being active below 0.25 were removed from the graph for clarity. For each diagram, the associated EEG measurements from which they were computed are also presented. The top panels illustrate the information projected back on the anatomy for short data windows. 